\title{
Congenital Perineal Groove Defect in Monozygotic Twin Infants: A Literature Review
}

\author{
Mimily Harsono, MD, $\mathrm{MSc}^{1,2}$ \\ David Yanishevski, MD² \\ ${ }^{1}$ Department of Pediatrics, Division of Neonatal-Perinatal Medicine, \\ University of Tennessee Health Science Center, Memphis, Tennessee \\ ${ }^{2}$ Department of Pediatrics, University of Tennessee Health Science \\ Center, Memphis, Tennessee \\ ${ }^{3}$ Department of Obstetrics/Gynecology, University of Tennessee \\ Health Science Center, Memphis, Tennessee \\ ${ }^{4}$ Department of Physiology, University of Tennessee Health Science \\ Center, Memphis, Tennessee
}

Massroor Pourcyrous, MD ${ }^{1,2,3,4}$

Address for correspondence Mimily Harsono, MD, MSc, Department of Pediatrics, Division of Neonatal-Perinatal Medicine, 853 Jefferson Avenue, Suite-201, Memphis, TN 38103

(e-mail: mharsono@uthsc.edu).

Am J Perinatol Rep 2021;11:e54-e57.

\begin{abstract}
Perineal groove is a rare benign congenital anomaly with lesion that resembles perforation of mid-perineum or perineal raphe area. Most reported cases of congenital perineal groove presented as an isolated defect in term or early-term singleton female infants. Thus far, there is no reported case of this anomaly in monozygotic twins. Embryo pathogenesis of this female predominance congenital defect remains controversial. Many clinicians are unfamiliar with this congenital anomaly. This congenital defect tends to get self-resolved at around 2 year of age. Nevertheless, the exposed nonepithelized mucous membrane can carry risk of local infection or irritation with the possibility of requiring early surgical correction. The defect can be infrequently associated with other ano-urogenital malformations that required immediate surgical intervention. Most isolated cases tend to be asymptomatic and self-healed with expectant management. Surgical correction may be considered if not healed after

\section{Keywords}

- congenital anomaly

- perineal groove

- perineal defect

- monozygotic twins

- newborn 2 years of age. Early diagnosis at birth is important to avoid misdiagnoses at later age for trauma, dermatitis, sexual abuse, and risk of unnecessary aggressive intervention. Early parental counseling for providing good hygiene and close follow-up is important to prevent infection or inflammation. Presentation of this anomaly in both monozygotic twins may support the hypothesis of potential disruption during embryo morphogenesis stages.
\end{abstract}

Congenital perineal groove is a rare female predominant defect of the perineum. The lesion looks like a perforation of the midline-perineal region. This congenital malformation resembles a failure of midline perineal fusion or midline perineal skin epithelization. ${ }^{1,2}$ The defect presents as an exposed wet nonepithelized erythematous mucous membrane that

received

December 28, 2020

accepted

January 11, 2021
DOI https://doi.org/ 10.1055/s-0041-1727145. ISSN 2157-6998. extends vertically from the anterior ridge of anal orifice toward the posterior edge of the vaginal fourchette. ${ }^{2,3}$ Nearly all of the reported cases of this congenital anomaly were discovered in term or near-term female infants. ${ }^{1,2,4-18}$ Thus far, there is no reported case of congenital perineal defect in both monozygotic monochorionic-diamniotic preterm twin infants.

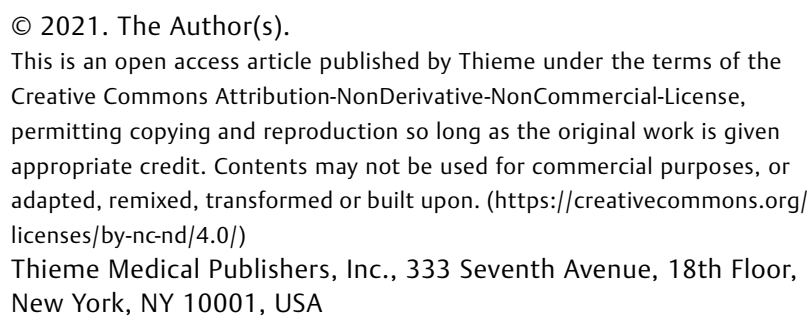




\section{Case Presentation}

A set of 34 weeks African American monochorionic-diamniotic female twins was delivered via normal vaginal birth. Twin A was with birth weight (BW) of $1,850 \mathrm{~g}$ and Apgar scores of 8 and 9 at 1 and 5 minutes, respectively. Twin $B$ was with BW of 2,020 g and Apgar scores of 8 and 9 at 1 and 5 minutes as well. Both twins received delayed cord clamping for 60 seconds and were admitted to neonatal intensive care unit (NICU) for premature infant's care. Mother was a 38year-old G8P6 with poor prenatal care. Mother received prenatal steroid, betamethasone, for 48 hours prior to delivery. No reported family history of congenital malformation was noted. Mother used cannabis during her pregnancy with cord-tissue toxicology screen positive for tetrahydrocannabinol. Mother did not have a significant major medical condition except for a history of treated Escherichia coli urinary tract infection a week prior to delivery. Placental pathology was normal on maternal side with fetal side for twin A suspected with acute chorioamnionitis and twin B suspected with focal early infarct. On admission and physical examination, a complete midline perineal defect was noted in twin A. The defect presented as an exposed wet groove or sulcus with nonkeratinized erythematous mucous membrane that stretched vertically from anterior rim of the anal opening to the posterior edge of the vaginal fourchette ( - Fig. 1). All other physical exams of twin A were normal including normal position of the urethra and vaginal orifices, and normal position of the anal opening. Surprisingly, we noted the same perineal defect during the physical exam of twin B (-Fig. 2). Both twins had normal bladder and rectal functions. Head ultrasounds showed bilateral grade-I subependymal hemorrhage on both twins. Echocardiograms were

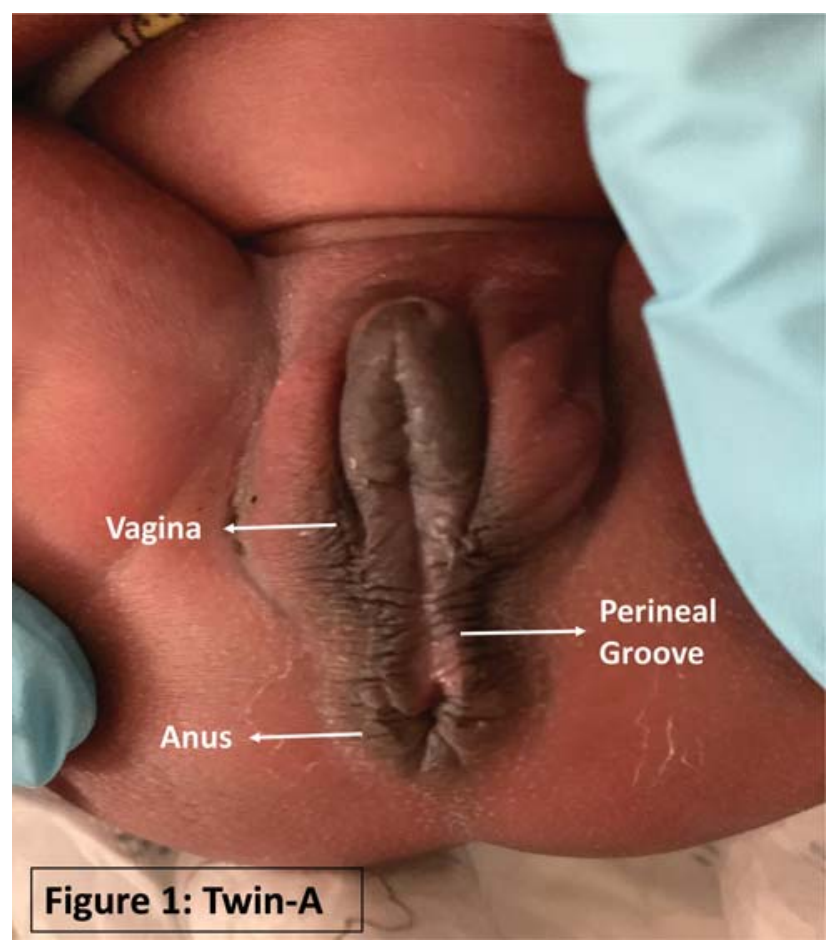

Fig. 1 Twin A. normal with normal newborn left to right shunting through patent foramen ovale on both twins. Renal and pelvic sonograms were normal for both twins as well. Both twins stayed in NICU for 3 weeks for their prematurity-associated oral feeding skill maturation. An improvement of the perineal defect was noted on twin B prior to discharge. Mother was educated on infection prevention of the area of defect and routine follow-up was encouraged.

\section{Discussion}

The true incidence rate of this congenital defect is unknown. The minor defect and self-resolved nature of this congenital anomaly make it easily missed at birth and under reported. Several case reports from across the globe have identified that this defect can affect any ethnicity and largely prevalent in female infants. ${ }^{2-18}$

Congenital perineal groove malformation appears as an exposed wet nonepithelized erythematous mucous membrane sulcus that extends vertically downward from the posterior portion of the vaginal fourchette to the anterior rim of anus. $2,3,11$ There are two forms of congenital perineal groove $^{13,15}$ : the complete form with the lesion extends from posterior vaginal fourchette to anterior rim of anus, and the incomplete form. The incomplete form may involve just the anterior part of the perineum, or the posterior part of the perineum. Two retrospective studies reported a complete form in $55 \%$ of their observed cases. Amongst their incomplete cases, posterior defect accounted for $30 \%{ }^{13,15}$ This congenital defect may infrequently be associated with other anatomical and/or functional malformation of the surrounding structures such as fecal continence/incontinence, anterior anus, prolapse anus, ectopic anus, and/or urinary tract defect. ${ }^{2,9}$

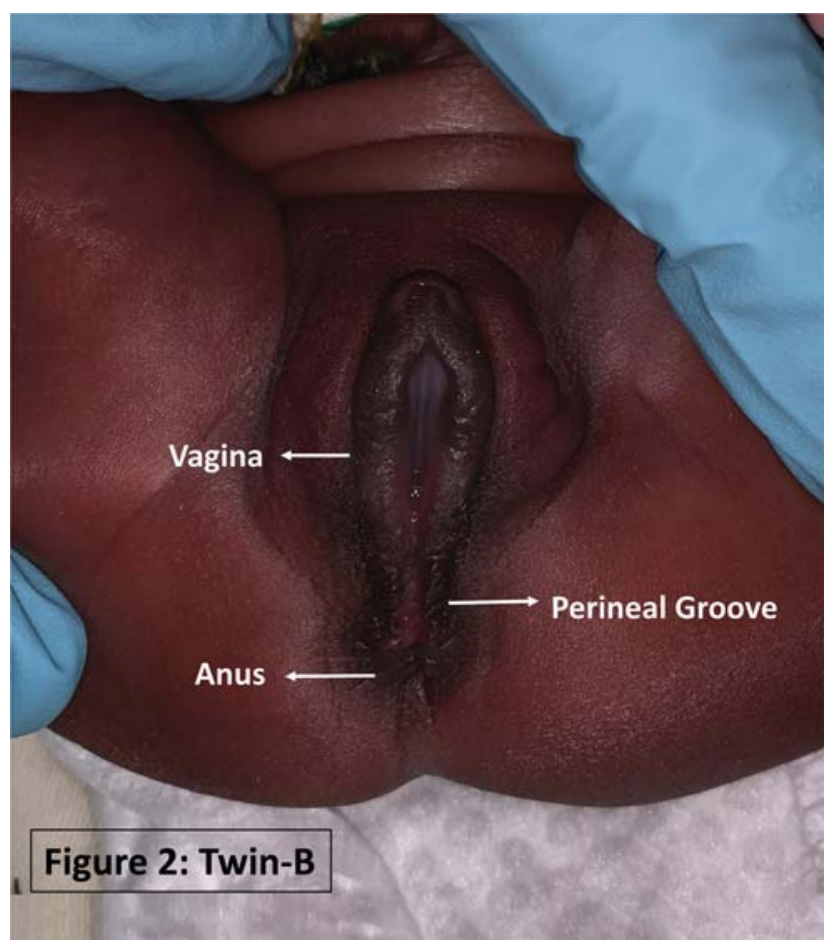

Fig. 2 Twin B. 


\section{Embryo Pathogenesis and Histology}

In 1968 , Stephens ${ }^{2}$ originally described perineal groove as a congenital malformation that consists of three primary features: "(1) a wet groove in mid-perineum between the fourchette and the anus; (2) normal formation of the vestibule including urethra and vagina; and (3) hypertrophy of the minoral tails that skirt the perineum and course posteriorly to join at the anus or to surround it."

Embryologically, the pathogenesis of congenital perineal groove remains controversial and poorly understood. It is thought to be associated with multifactorial influences during embryology developmental phases. Several embryopathy mechanisms have been hypothesized, which are: (1) an open cloacal duct remnant, ${ }^{19}$ (2) urorectal septum developmental defect during cloacal embryological stages at 4 th to 8 th week of gestational age, ${ }^{20,21}$ (3) midline fusion failure of the medial genital folds between the perineal raphe and the vestibule, ${ }^{2,22,23}$ (4) disruption of Sonic hedgehog (Shh) signaling expression and its derivative pathway genes, ${ }^{24-26}$ (5) exposure to teratogenic substance or disruption of blood supply during embryogenesis or morphogenesis. $21,24,25,27,28$

With limited number of reported male cases, ${ }^{13,15,23}$ an embryologic disruption of external genitalia developmental phases has been considered as one of the potential hypotheses. External genitalia of both sexes arise from the genital tubercle, genital folds, and labioscrotal folds. Failure of labioscrotal folds fusion to form perineal raphe will cause perineal grove to occur. ${ }^{1,22}$ Histologic evaluation of the resected lesions varied from a nonkeratinized stratified squamous epithelium without sebaceous glands or sweat glands or hair follicles, ${ }^{2,22,25,26}$ to a simple columnar or stratified columnar or cuboidal epithelium of a rectal type mucosa with intervening area of a nonkeratinized stratified squamous epithelium. ${ }^{20}$ This histological portion resembled an anorectal transition zone epithelium..$^{20}$ This finding suggests that congenital perineal grove malformation is probably associated with defect during urorectal septum development of cloacal embryology stages. 1,20,21,26,29

\section{Embryo Molecular and Morphogenesis}

Embryo molecular and morphogenesis studies quoted the potential role of Shh signaling expression and its downstream mediators, Gli2, Gli3, Hoxa-13, and Hoxd-13, during early cloacal embryogenesis differentiation into dorsal anorectum canal and ventral urogenital sinus. ${ }^{24,25,27}$ Urorectal septum formed from primitive urogenital sinus and primitive hindgut. ${ }^{30} \mathrm{Shh}$ is expressed in cloacal endoderm and hindgut endoderm cells, and signaling development of urorectal septum and caudal hindgut in perineum morphogenesis. ${ }^{24,25}$ Studies of Shh co-regulatory signaling gene, bone morphologic protein 4, and its down regulator gene Fgf10 and Ptch1, demonstrated their possible involvement in anourogenital epithelization morphogenesis. ${ }^{27-29}$ Animal studies showed that exposure to teratogenic substances will disrupt gene signaling expression during embryogenesis. We assume that the presentation of congenital perineal groove in our monozygotic twins may be a sign of an association with the disruption of the molecular signaling genes during cloacal embryogenesis stages.

\section{Diagnosis}

Congenital perineal groove is a clinical diagnose. The nature of its subtle defect and potential for spontaneous self-resolution, make this defect easily missed during newborn physical exam. Early diagnosis of this defect at birth is important, as this lesion can resemble rupture or inflammation of the perineum area, ${ }^{8,11}$ and failure to recognize this defect at birth will lead to misdiagnosis at later age for traumatic injury, irritant dermatitis, diaper rash, infection, lichen sclerosis, ulcerated hemangioma, anal fissure or sexual abuse. ${ }^{8,17}$ ARM-net consortium questionnaire study in January 2019 reported that only $9 \%$ of congenital perineal groove were identified within the first week of life by neonatologist in training centers. ${ }^{15}$ Infrequent association with anomalies of ano-urogenital organs or other major organs, makes it necessary to screen for anomaly of the surrounding structures, ${ }^{9,15}$ such as perineal cutaneous fistula which is a surgical emergency. $3,13,15$ If misdiagnosed, this benign self-resolved malformation can lead to unnecessary treatment or intervention including topical steroid, topical antifungal, skin biopsy, laser therapy, etc. ${ }^{8-10,17}$

\section{Management and Complication}

Congenital perineal groove tends to be self-resolved or selfepithelized at around 2 years of age, and in some reported cases it may take as long as 4 years of age. ${ }^{1,3,8,13,15}$ Majority of the isolated cases are asymptomatic with no other associated anomaly and with normal ano-urogenital function. Asymptomatic cases tend to heal naturally. Large cohort retrospective observation studies reported that close to $75 \%$ of the cases were self-resolved. ${ }^{13,15}$ Conservative or expectant management is the practice of choice. However, patients with this nonepithelized mucous membrane are at risk of infection, inflammation, urinary tract infection, fecal incontinence, constipation, and functional or structural anomaly of the surrounding ano-urogenital organs. ${ }^{9,15,17}$ Surgical correction can be performed for cosmetic reasons if the lesion is not epithelized after 2 years. Persistent inflammation or infection to the nonepithelized lesion from mucous drainage and wet secretion from the urethra, vaginal, or anal sites, would be a clinical indication for surgical intervention. ${ }^{8,13,15}$ Most common complication of perineal groove repair surgery is wound dehiscence and/or wound infection. Early parental counseling on hygiene and gentle care of the isolated defect is essential in preventing unnecessary infection and irritation.

\section{Conclusion}

Perineal groove is a rare benign self-resolved congenital malformation. The defect tends to be isolated, asymptomatic, female predominant, and self-epithelialized at 2 years of age. Early diagnosis of this congenital defect at birth is important to avoid misdiagnosis at a later age for trauma, dermatitis, or even sexual abuse and unnecessary aggressive surgical or 
medical intervention. With the potential for natural healing, this defect is managed conservatively. It is important to provide early parental counseling on hygiene, gentle care, infection or irritation precautions, and a good follow-up care. Surgical intervention is required for recurrent infection or irritation, nonepithelialization after 2 years of age, and cosmetic reasons. Presentation of this anomaly in monozygotic twins may support the hypothesis of potential disruption during the perineal embryo molecular and morphogenesis stages.

\section{Authors' Contribution}

M.H. was involved in diagnosis, patient care, literature review, obtaining IRB approval, obtaining parental consent, photographs, writing the first manuscript draft, revision, and approval of the final manuscript as submitted. D.Y. was involved in diagnosis, patient care, literature review, writing the first draft of the case presentation, and approval of the final manuscript as submitted. M.P. was involved in diagnosis, patient care, literature review, critical review, revision, and approval of the final manuscript as submitted.

\section{Financial Disclosure}

The authors and/or their family members do not have any financial association relevant to this article to disclose.

\section{Patient Consent}

The case report study, which included the photographs have received an IRB approval from University of Tennessee Health Science Center and Regional One Health Hospital to conduct the study. All photographs and clinical history provided in this case report have a written consent form signed by both twin patients' parent or legal guardian for the purpose of medical literature publication and education. Both patients' personal information was deidentified in the manuscript article and figures.

\section{Funding Source \\ No external funding.}

\section{Conflict of Interest}

The authors and/or their family members do not have any potential conflict of interest to disclose.

\section{References}

1 Sekaran P, Shawis R. Perineal groove: a rare congenital abnormality of failure of fusion of the perineal raphe and discussion of its embryological origin. Clin Anat 2009;22(07):823-825

2 Stephens FD. The female anus, perineum and vestibule. Embryogenesis and deformities. Aust N Z J Obstet Gynaecol 1968;8(02): 55-73

3 Kadowaki H, Nakahira M, Yamada C, Takeuchi S, Tamate S, Shiokawa C. Perineal groove and perineal canal. Jpn J Surg 1983;13(03):216-218

4 Barbosa M, Alves N, Fontes N. Perineal groove: a rare congenital anomaly. Acta Med Port 2016;29(10):670-672

5 Boutsikou T, Mougiou V, Sokou R, et al. Four cases of perineal groove-experience of a Greek Maternity Hospital. Medicina (Kaunas) 2019;55(08):E488
6 Cheng H, Wang Z, Zhao Q, Zhu H, Xu T. Perineal groove: report of two cases and review of the literature. Front Pediatr 2018;6:227

7 de La Monneraye Y, Benoist G, Marchesi L, Crosnier AS, GuinardSamuel V. Perineal groove: case report and review of the literature. Arch Pediatr 2017;24(12):1259-1261

8 Diaz L, Levy ML, Kalajian A, Metry D. Perineal groove: a report of 2 cases. JAMA Dermatol 2014;150(01):101-102

9 Esposito C, Giurin I, Savanelli A, Alicchio F, Settimi A. Current trends in the management of pediatric patients with perineal groove. J Pediatr Adolesc Gynecol 2011;24(05):263-265

10 Garcia-Palacios M, Mendez-Gallart R, Cortizo-Vazquez J, Rodriguez-Barca P, Estevez-Martinez E, Bautista-Casasnovas A. Perineal groove in female infants: a case series and literature review. Pediatr Dermatol 2017;34(06):677-680

11 Harsono M, Pourcyrous M. Perineal groove: a rare congenital midline defect of perineum. AJP Rep 2016;6(01):e30-e32

12 Hunt L, Srinivas G. Newborn with a perineal lesion. Pediatr Rev 2016;37(01):e1-e3

13 Ihn K, Na Y, Ho IG, Oh JT. Clinical characteristics and conservative treatment of perineal groove. J Pediatr Surg 2019;55(08): 1507-1510

14 Pastene SC, Rojas MF. Perineal groove in pediatric gynecology: a report of 2 cases. Rev Chil Pediatr 2014;85(04):486-490

15 Samuk I, Amerstorfer EE, Fanjul M, et al. Perineal groove: an anorectal malformation network, consortium study. J Pediatr 2020;222:207-212

16 Siruguppa K, Tuli SS, Kelly MN, Tuli SY. Newborn female with a midline perineal defect. Clin Pediatr (Phila) 2012;51(02):188-190

17 Verma SB, Wollina U. Perineal groove-a case report. Pediatr Dermatol 2010;27(06):626-627

18 Wojciechowski M, Van Mechelen K, Van Laere D. Congenital perineal groove. Arch Dis Child 2019;104(03):286

19 van der Putte SC. Normal and abnormal development of the anorectum. J Pediatr Surg 1986;21(05):434-440

20 Mullassery D, Turnock R, Kokai G. Perineal groove. J Pediatr Surg 2006;41(03):e41-e43

21 Stephens FD. Embryology of the cloaca and embryogenesis of anorectal malformations. Birth Defects Orig Artic Ser 1988;24 (04):177-209

22 Abdel Aleem A, el Sheikh S, Mokhtar A, Ghafouri H, Saleem M. The perineal groove and canal in males and females-a third look. Z Kinderchir 1985;40(05):303-307

23 Chatterjee SK, Chatterjee US, Chatterjee U. Perineal groove with penoscrotal hypospadias. Pediatr Surg Int 2003;19(07):554-556

24 Runck LA, Method A, Bischoff A, et al. Defining the molecular pathologies in cloaca malformation: similarities between mouse and human. Dis Model Mech 2014;7(04):483-493

25 Seifert AW, Harfe BD, Cohn MJ. Cell lineage analysis demonstrates an endodermal origin of the distal urethra and perineum. Dev Biol 2008;318(01):143-152

26 van der Putte SC. The development of the perineum in the human. A comprehensive histological study with a special reference to the role of the stromal components. Adv Anat Embryol Cell Biol 2005; $177: 1-131$

27 Haraguchi R, Mo R, Hui C, et al. Unique functions of Sonic hedgehog signaling during external genitalia development. Development 2001;128(21):4241-4250

28 Roberts DJ, Smith DM, Goff DJ, Tabin CJ. Epithelial-mesenchymal signaling during the regionalization of the chick gut. Development 1998;125(15):2791-2801

29 Jin ZW, Jin Y, Li XW, Murakami G, Rodríguez-Vázquez JF, Wilting J. Perineal raphe with special reference to its extension to the anus: a histological study using human fetuses. Anat Cell Biol 2016;49 (02):116-124

30 Hynes PJ, Fraher JP. The development of the male genitourinary system. I. The origin of the urorectal septum and the formation of the perineum. Br J Plast Surg 2004;57(01):27-36 